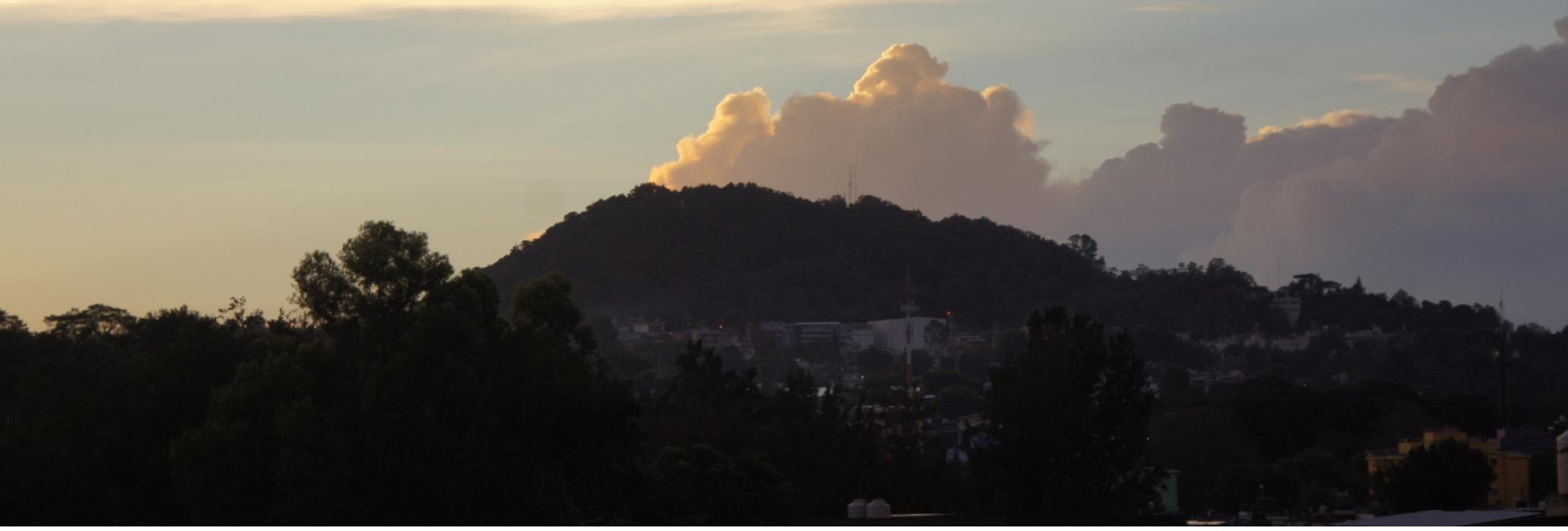

\title{
Análisis del crecimiento económico y desarrollo humano de las entidades federativas de México en el periodo 2005 - 2010
}

\section{F. Beltrán - Guerra1 - J. L. Arellanez - Hernández - E. Romero - Pedraza}

RESUMEN: El gasto público en México se orienta a crear condiciones de desarrollo humano, salud y bienestar social por parte del Estado a través de su estructura federal. Se busca identificar cuál es el comportamiento de los indicadores de crecimiento y desarrollo de las entidades federativas en México en el periodo comprendido de 2005 a 2010. Con el análisis estadístico, se concluye que la relación entre el gasto público, destinado a rubros de salud, educación, desarrollo de infraestructura social, tiene una baja correlación con los índices de desarrollo en el país. Las variaciones no son significativas pero si las diferencias entre entidades federativas. El análisis de los indicadores mencionados, pretende mostrar que el gasto público no impacta claramente en las condiciones de salud, desarrollo y bienestar social de los mexicanos, elevando su calidad de vida.

Palabras Clave: Gasto público, Indice de Desarrollo Humano, Bienestar Psicológico, Calidad de vida, Condiciones de vida.
ABSTRACT: Public spending in Mexico aims to create conditions of human development, health and social well-being by the State through its federal structure. Our target is to identify what is the behavior of the indicators of growth and development in Mexico in the period of 2005-2010. After the statistical analysis, it is concluded that the relationship between public spending aimed at areas of health, education, social infrastructure development, has a low correlation with development index in the country. The variations in this index are not significant in the years analysed, but the differences between states does. The proposal aims at the public spending for being reflected significantly in the conditions of health, development and welbeing of Mexican people.

Keywords: Public expenditure, Human development Index, Psychological well-being, Quality of life, Life conditions.

1 Universidad Veracruzana. Instituto de Investigaciones Psicológicas. Observatorio de Calidad de Vida y Salud Social. Correo:lebeltran@uv.mx 
Introducción

$\perp$ as necesidades sociales que debe satisfacer el Estado, no se cubren con el simple hecho de proporcionar dinero, la realidad humana es mas compleja. "Las necesidades públicas son aquellas que los habitantes sienten o deberían sentir como miembros de la comunidad política y que son satisfechas con el gasto público, aunque no sean advertidas por muchos de los miembros que contribuyen con las cargas públicas". (Faya, 2008, p. 12)

De acuerdo con Faya en 2008, el cual citando a Fonrouge destaca que hoy día, "no hay correlación entre necesidades individuales y colectivas, ni estas son la suma de aquellas, aparte de que al efectuar el Estado una selección de necesidades, el principio hedonista no resulta absoluto" (Fayya, 2008, p. 11) De manera específica, uno de los problemas sociales que demandan una intervención clara es la medición del progreso y bienestar, para impactar en las decisiones financieras de este rubro favoreciendo la actuación del Estado en crear condiciones para elevar la calidad de vida de los mexicanos.

Particularmente, en los últimos años el debate entre crecimiento y desarrollo de los países, ha integrado específicamente en el centro de la discusión, a los individuos y cómo estos conciben que su vida se ha visto impactada mas allá de los indicadores económicos, mas allá de los hechos (Lora, 2008). Los estudios realizados a nivel Latinoamérica en esta línea de investigación, llevan a plantear interrogantes en torno a cómo conceptualizar el progreso mas allá del producto interno bruto (PIB) como eje de mediciones y decisiones, qué se debe medir, y cómo utilizar dichas mediciones para la toma de decisiones en políticas públicas. (Rojas 2011a; Rojas, 2011b; Rojas 2012). Es así que surgen las siguientes interrogantes: ¿cómo impactan las acciones del gobierno y las políticas del Estado en la vida de las personas, en su satisfacción, en el bienestar, en su calidad de vida?, pero sobre todo, ¿cuál es la relación de dicho impacto con las necesidades sociales?

El Programa de las Naciones Unidas para el Desarrollo en 2011 (PNUD, 2011), en su área de trabajo relativa al Desarrollo Humano, destaca la necesidad de las naciones de trascender del crecimiento económico, orientando al desarrollo de los individuos, los cuales puedan elegir una vida donde puedan realizar con plenitud su potencial como seres humanos. A su vez, conceptualiza al Desarrollo Humano como la posibilidad de los individuos para elegir con libertad una vida larga y saludable, en condiciones de poder acceder a oportunidades de obtener los recursos necesarios para vivir decorosamente.

De manera paralela, en 2009, se publica el Informe de la Comisión para la Medición del Desempeño Económico y Progreso Social, encabezado por Zen, Stiglitz, Fitoussi (Sen, Stiglitz, Fitoussi, 2009), donde como primer punto, resaltan cómo la medición del progreso y bienestar se ha visto limitado al considerar solamente el producto interno bruto (PIB) y derivar de ello las mediciones pertinentes para la toma de decisiones, reconocimiento que, dadas las características sociales, culturales, políticas, económicas de los fenómenos derivados de las finanzas públicas, se requiere incluir otras variables que conlleven a generar alternativas para la medición del progreso social, así como herramientas diferentes.

Dicho estudio, propone que es posible encontrar diversas explicaciones para la diferencia entre la medición estadística de los fenómenos socioeconómicos y la percepción ciudadana de los mismos fenómenos, donde los conceptos estadísticos pueden ser correctos, pero el proceso de medición puede ser imperfecto en la medición del progreso y bienestar bajo esta perspectiva. Sin embargo, la idea de que las formas de medición utilizadas afectan las formas de tomar decisiones, así como la ejecución de dichas acciones. Si las mediciones son defectuosas, las decisiones se distorsionan (Sen et al, 2009).

Hablando específicamente del caso de México y su participación en esta perspectiva de medición del progreso y bienestar, tomando como centro la perspectiva de los individuos paralela a los indicadores económicos ya establecidos, el Instituto Nacional de Estadística y Geografía (INEGI), atendiendo a las políticas y recomendaciones de la Organización para la Cooperación y el Desarrollo Económico 
(OCDE), el Informe de la Comisión para la Medición del Desempeño Económico y Progreso Social, así como del Programa de las Naciones Unidas para el Desarrollo (PNUD), se plantea la necesidad de iniciar procesos de medición integral que consideren los siguientes puntos: progreso material; calidad de vida a partir de indicadores objetivos y subjetivos; indicadores de sostenibilidad inter - generacional del progreso social. El bienestar subjetivo es uno de los varios elementos a considerar para una mejor medición del bienestar y el progreso social (INEGI, 2012). Se destaca la necesidad de contar con información relacionada con el nivel de bienestar en nuestro país, desde la perspectiva de la gente con relación a las leyes y operaciones que el Estado realiza con el dinero público, así como de su actuación, ya que tales percepciones influyen determinantemente en el comportamiento social y en cómo se percibe el bienestar y la calidad de vida.

\section{Método}

Se busca identificar cuál es el comportamiento de los indicadores de crecimiento y desarrollo en México, a partir de un Análisis de Componentes Principales (ACP), en un periodo comprendido del año 2005 al 2010 considerando las 32 entidades federativas del país como unidades de estudio. Las variables para este estudio son, el Índice de Desarrollo Humano (IDH) (PNUD, 2012a), así como el Índice de Competitividad Social (ICS) (PNUD, 2012b), establecidos por el Programa de las Naciones Unidas para el Desarrollo. Se consideran la densidad poblacional (DP) y el producto interno bruto (PIB) por entidad federativa, obtenidos del Conteo de Población y Vivienda de los años 2005 y 2010, así como del Banco de Información Económica del Instituto Nacional de Estadística, Geografía e Informática (INEGI). Finalmente, se considera el gasto púbico programado para el Ramo 033, en el Presupuesto de Egresos de la Federación (Observatorio de Política Social y Derechos Humano, 2011) en el mismo periodo, con pesos constantes. Con estos datos se construyó una base de datos tipo panel de 32 entidades federativas en 6 años consecutivos 2005-2010.

Para cada año se estudia el comportamiento de cada variable a partir de histogramas y gráficas de cajas que nos permitió identificar entidades atípicas; se estudió la evolución de cada variable en el tiempo, posteriormente se obtuvieron las correlaciones de Pearson y se realizan gráficos de dispersión de parejas de variables; para estudiar los grados de homogeneidad y variabilidad entre las entidades se corrió un análisis cluster por año, utilizando la distancia euclidiana y los métodos de aglomeración de "ward"; a partir de este análisis se clasificó a las entidades federativas en los grupos que se forman en términos de desarrollo y crecimiento en México. Estos análisis se consideraron de manera comparativa para identificar la evolución en el periodo de tiempo. Así mismo, se corrió un análisis de componentes principales para cada año y se realizó la interpretación de los dos primeros componentes. Posteriormente se consideraron las 32 entidades replicadas seis veces (una por cada año) y se corrió e interpretó un análisis de componentes principales de los datos en todos los años. Con base en ello, se realizó un análisis de componentes de la varianza sobre los dos primeros componentes principales. Finalmente, se realizó un análisis de regresión simple entre el gasto acumulado 2005 - 2010 para evaluar el impacto sobre el IDH para el año 2010.

\section{Resultados}

Al describir la distribución en Producto Interno Bruto (PIB) por año tomando en consideración las 32 entidades federativas, es posible apreciar entidades "outliners" que salen del conjunto de las entidades: el Distrito Federal, Estado de México y Nuevo León. Se destaca que para el año 2010, se incrementa el PIB y con ello la variación entre los estados. Figura 1.

En el análisis de la relación entre el tamaño de la población y el gasto público en el Ramo 033, el cual considera aportaciones a los estados con sus diversos fondos de desarrollo en salud, educación, e infraestructura en desa- 


\section{Figura 1. Gráfico de cajas del PIB en el periodo 2005 - 2010.}

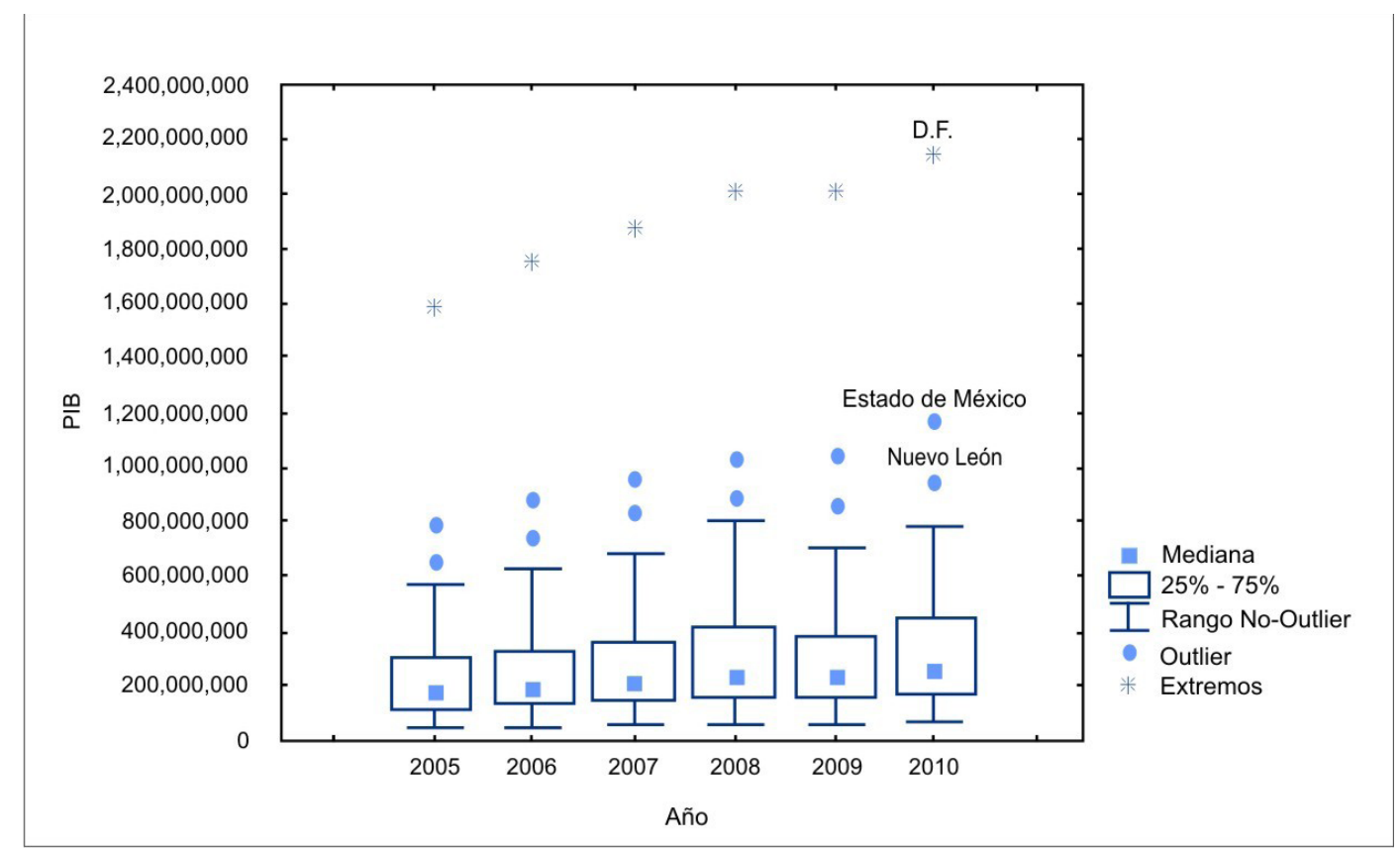

Elaboración propia con base en datos de INEGI: Sistema de Cuentas Nacionales de México. Cuentas nacionales > Producto interno bruto por entidad federativa > Por grupos de actividad económica > A precios corrientes $>$ Valores absolutos > Total de la actividad económica. Unidad de medida: Miles de pesos corrientes a precios básicos.

rrollo social, es posible destacar que existe una correlación de $\mathrm{r}=0.72$ para el año 2005, y de $\mathrm{r}=0.73$ para el 2010 (el nivel de confiabilidad para esta correlación fue de 0,05). Sin embargo, se destaca que en el caso del Distrito Federal, aun cuando tiene una densidad poblacional alta, esta no se encuentra en proporción con el recurso del gasto público destinado a crear condiciones de bienestar y desarrollo.

Dado que en el análisis de las variables por entidad federativa en los años de 2005 a 2010 no muestran variaciones significativas, se decidió centrar dicho análisis de parejas de variables en 2005 y 2010, mostrando condiciones muy similares en el periodo de tiempo establecido. Figura 2.

Con base en el análisis realizado y con la intención de identificar las agrupaciones que se forman considerando las entidades federativas, así como las variables: índice de desarrollo humano, índice de competitividad social, población, producto interno bruto, y gasto público, a partir de un análisis cluster con el método de Wards y utilizando distancias euclideanas, para el año 2005 se identificaron cuatro gran- des grupos, sin embargo, para el 2010, se identifican tres grupos, lo que puede interpretarse como que al paso de los años analizados, mas que impactar en el incremento en los valores de cada variable, se ha logrado homogeneizar las condiciones entre las diversas entidades federativas, particularmente en el caso de Tabasco, Guanajuato, Veracruz, Campeche, Puebla, Tamaulipas, Chihuahua, Sonora, Coahuila, Michoacán, y Baja California. Sin embargo, los estados con menores puntuaciones en los indicadores, tienen el mismo efecto, integrándose en este grupo: Sinaloa, San Luis Potosí, Queretaro, Chiapas, Oaxaca, Hidalgo, Yucatán, QuitanaRoo, Guerrero, Zacatecas, Durango, Morelos y Aguasalientes. Figura 3.

Al correr el análisis de componentes principales (ACP) en el año 2005, se encontró que los dos primeros componentes explicaron el 92\% de la varianza total; en el 2010, este porcentaje sólo tuvo un incremento a $92.5 \%$.

Al revisar los pesos de estos componentes para el año 2005, el componente principal 1 (CP1) obtuvo 0.35 de IDH, 0.32 de ICS, y 0.24 de Ramo033, lo que nos lleva a identificarlo 


\section{Figura 2. Correlación de indicadores de desarrollo y crecimiento en 2005 y 2010.}

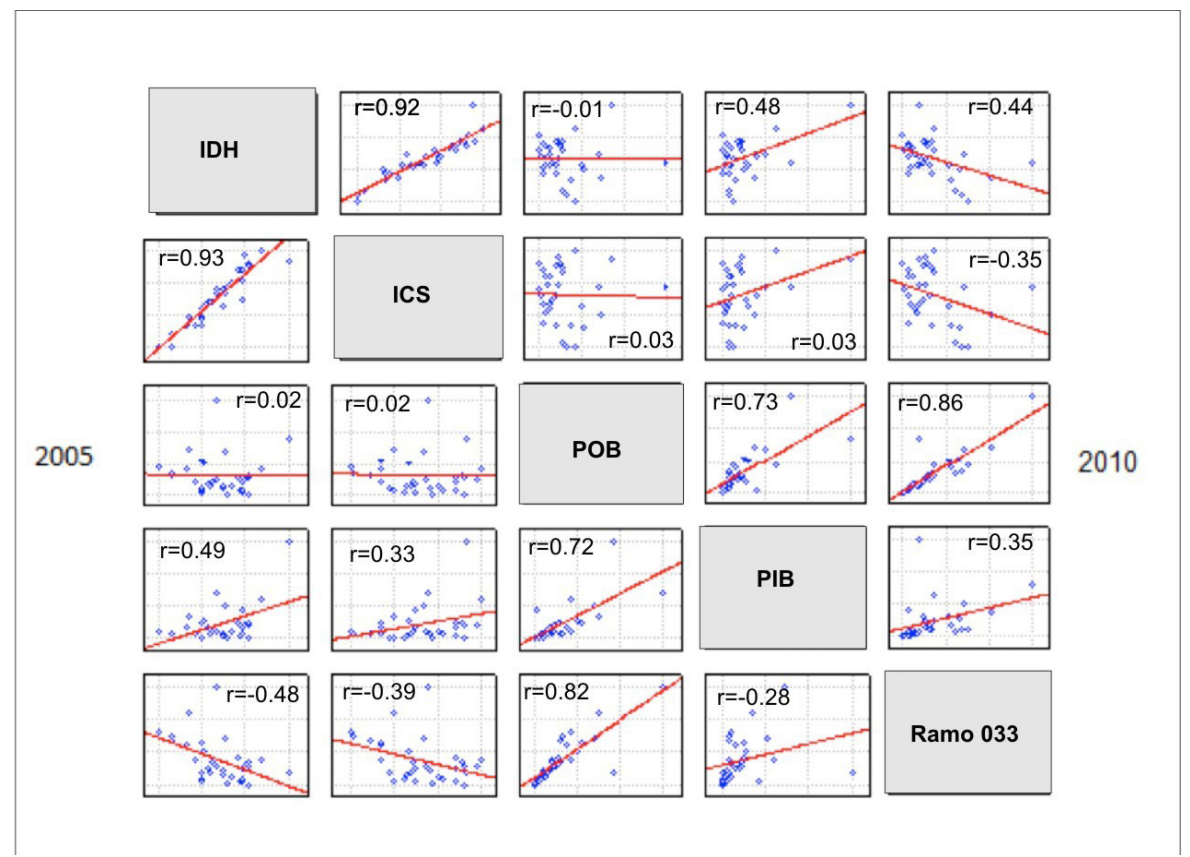

Elaboración propia con base en datos obtenidos de OCDE 2010 para IDH e ICS; datos del Censo de Población y Vivienda INEGI 2005 y 2010; Presupuesto de Egresos de la Federación para el gasto público con datos de Observatorio de Política Social 2013.

Figura 3. Análisis Cluster comparando agrupaciones Superior 2005 y Inferior 2010.

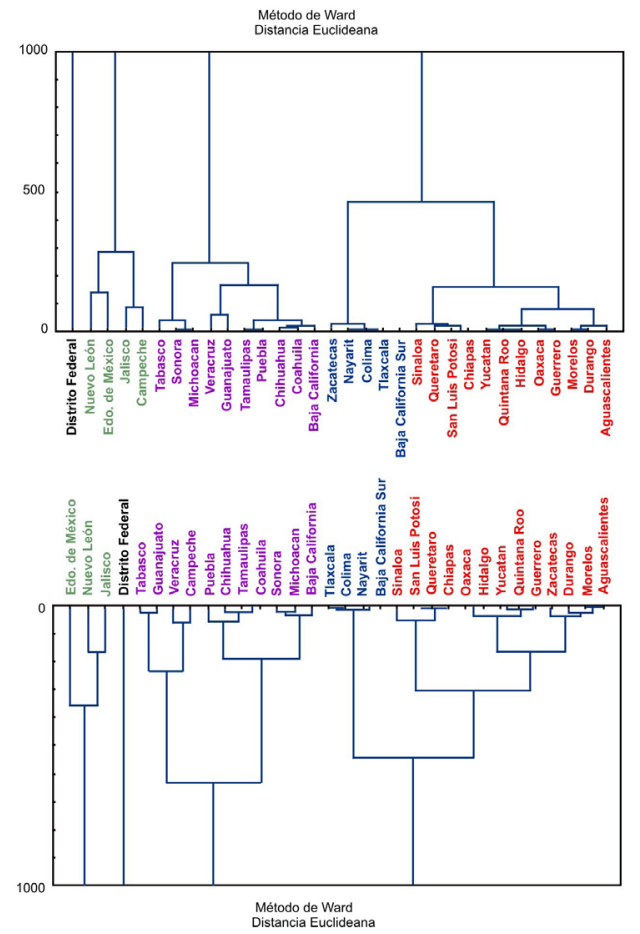

Elaboración propia con base en datos obtenidos de OCDE 2010 para IDH e ICS; datos del Censo de Población y Vivienda INEGI 2005 y 2010; Presupuesto de Egresos de la Federación para el gasto público con datos de Observatorio de Política Social 2013. 
como "Componente de Desarrollo"; a su vez, el componente principal 2 (CP2) para el mismo año obtuvo 0.37 de POB (población) y 0.36 en PIB, lo que hace posible identificarlo como "Componente de crecimiento". Para el 2005, las entidades con valores positivos en el CP1 son el Distrito Federal y Nuevo León. Se destaca a su vez que para el caso de Veracruz y el Estado de México, se presentan con valores negativos en ambos componentes, es decir, que no se aprecian valores característicos del componente de desarrollo ni de crecimiento. De igual forma, se destaca que Chiapas, Oaxaca, Guerrero y Michoacán, se ubican con valores negativos en el $\mathrm{CP} 1$, y con valores cercanos a 1 en el CP2, lo que refleja que son entidades que se caracterizan por el componente de crecimiento de manera favorable, pero con efectos negativos en el componente de desarrollo.

Para el análisis de componentes principales del año 2010, los pesos se presentan de la siguiente forma: el componente principal 1 (CP1) obtuvo 0.35 de Ramo033, 0.21 de IDH, y 0.21 de ICS, lo que permite identificarlo igualmente como "Componente de Desarrollo"; para el componente principal 2 (CP2) de ese año, se compone con 0.35 de PIB, 0.21 de IDH y 0.20 en POB (población), identificándolo esta vez como "Componente de crecimiento y desarro1lo". Aun cuando el CP2 se integra la variable de IDH en 2010, las entidades antes mencionadas no cambian su posición. Para confirmar tal situación, se realizó un análisis de componentes principales una vez mas con los valores promedios para cada entidad por año, destacando que al paso del tiempo, el CP1 explica el 47\% de la varianza total, y el CP2 explica el 45\%, con los siguientes pesos: CP1 0.31 de IDH, 0.29 de ICS, y 0.28 de Ramo033; CP2 0.37 de PIB (población) y 0.32 en POB.

Ante tales resultados, fue conveniente analizar el impacto del gasto público acumulado de 2005 a 2010 relacionado con el IDH por entidad federativa, teniendo como resultado un coeficiente de correlación de pearson de -0.45. El mismo análisis en un modelo de regresión lineal simple, teniendo como variable respuesta el gasto público, los resultados fueron los siguientes:

\section{Gasto Públıco $_{i}=593958-631192$ IDH}

A partir de tales resultados, es posible confirmar que, el gasto público del Ramo 033 destinado para que las entidades federativas incidan en los indicadores de desarrollo humano de manera favorable es negativo El gasto público no ha modificaco los indicadores de bienestar. Para concluir con los resultados, a partir de un análisis de componentes de la varianza, tomando como base los valores del CP1 para cada entidad por año, para probar que esta no es significativa.

El valor obtenido de componentes de la varianza es de 1.02 con un p - valor de 0.001 lo que muestra que si existen variaciones entre las entidades federativas. Sin embargo, al analizar los años, el valor de componentes de la varianza obtenido es de -0.03 con un p-valor de 0,99, lo que nos lleva a identificar que no existen variaciones entre dichos años, es decir, que el gasto ejercido no ha generado cambios significativos en las condiciones de las entidades federativas cuando se consideran las variables de desarrollo humano, competitividad social, crecimiento económico y gasto público.

\section{Conclusiones}

En el gasto público destinado para las estindades federativas con fines de crear condiciones de desarrollo humano y bienestar social, se considera entre otros elementos, para sus reglas distribución y operación, la densidad poblacional, presentándose este elemento con mayor correlación por encima de los indicadores de desarrollo humano o de competitivodad social.

Ante tal situación es posible considerar que dichos indicadores establecidos por la OCDE, no solo muestran una baja correlación, sino que a su vez no son considerados para la toma de decisiones financieras que permitan impactar favorablemente en el incremento de los índices de desarrollo humano.

Tal situación cuestiona las formas en que se toman las decisiones sobre el gasto público y 
las políticas públicas que lo acompañan, pues el dinero se destina sin percibir un impacto significativo en los resultados de las condiciones de calidad de vida de las personas. Es necesario destacar que aun cuando el análisis no muestra un impacto significativo en los indicadores de desarrollo, es posible apreciar que en los seis años analizados las características de las entidades federativas tienden a homogeneizarse para el 2010.

De igual forma, el análisis de componentes principales permite identificar que hay entidades en el país que aun cuando hay un esfuerzo por incrementar los indicadores de crecimiento, estos no tienen una respuesta positiva en el desarrollo, es decir, que aun cuando se destinan recursos públicos para el gasto en salud, educación y desarrollo social, estos no incrementan las condiciones de desarrollo humano y bienestar en la población, como es el caso de Chiapas y Oaxaca. A partir de este análisis es que se puede afirmar que el gasto público no necesariamente ha impactado favorablemente en el bienestar de la gente.

El análisis de regresión lineal simple, permite confirmar que el monto acumulado del gasto público en el Ramo 033 para los años 2005 a 2010, tiene una correlación negativa, lo que nos lleva a cuestionar las formas en que se toman las decisiones financieras para generar condiciones de desarrollo y bienestar social, así como las formas de medición de desarrollo en México y el impacto que se obtiene. De igual forma, el análisis de componentes de la varianza realizado, evidencia que existen diferencias significativas en las condiciones de las entidades federativas en el país, pero se resalta que, al realizar el análisis por años, no existen variaciones significativas.

En el periodo comprendido de 2005 a 2010, el gasto público ha servido para mantener las condiciones de desarrollo humano en el país, pero no para incrementarlas, lo que lleva a cuestionar las formas en que se toma las decisiones sobre el gasto público así como las fuentes de información con que se sustentan dichas decisiones. Al parecer los indicadores macroeconómico ya no son suficientes y se requiere complementar obteniendo información desde los destinatarios, desde la gente, con la intensión de relacionar los resultados con la realidad de la gente.

A manera de consideraciones finales, es posible resaltar que el caso de México como país, en relación a sus finanzas públicas, implica como siempre una serie de retos que permitan de manera equitativa, profesionalizar no solo al Estado, sino también a la academia y la sociedad, generando y aplicando conocimiento científico desde una perspectiva multidisciplinar. En lo que a las finanzas públicas de este país refiere, estas deberán estar adecuadas siempre al momento histórico y contexto en el que nos desenvolvemos, sin perder de vista las implicaciones que los procesos globales traen consigo.

Así mismo, es importante tener en consideración que las condiciones socioculturales consecuencia de las relaciones políticas, sociales, económicas y jurídicas muestran inevitablemente que tanto lo público como lo privado impacta de manera significativa en el desarrollo social, la justicia y la equidad. "(...) los que intentan guiar la economía y nuestras sociedades son como pilotos que intentan dirigir un rumbo sin una brújula confiable. Las decisiones que ellos (y nosotros, como ciudadanos individuales) tomamos dependen de lo que medimos, qué tan buenas son nuestras mediciones y qué tan bien se entienden nuestras medidas." (Sen et al, 2009, p. 9)

Se ha mostrado que la relación del gasto público destinado a rubros de salud, educación, desarrollo de infraestructura social, tiene una baja correlación con los índices de desarrollo en el país, y de igual forma, se evidencia que las variaciones entre años no son significativas, pero las diferencias entre entidades federativas sobresalen. Es por ello que se propone a partir del análisis planteado, que las mediciones del crecimiento y desarrollo económico en México, consideren otras variables de bienestar que permitan identificar si el gasto público se ve reflejado significativamente en las condiciones de salud, desarrollo y bienestar social de los mexicanos, elevando su calidad de vida.

La medición del progreso y bienestar considerando entre sus variables al bienestar como 
propuesta para dicha problemática, no debe ser solo una política establecida por los organismos internacionales o por un gobierno, son una necesidad demandada por los individuos no solo al Estado, sino también a la academia. De manera específica en nuestro país, aun hay mucho trabajo por hacer, la generación de información sigue considerándose una de las principales claves para encaminar el rumbo de un país en la búsqueda del bienestar, el progreso, la justicia social, la calidad de vida.

\section{Referencias}

Faya Viesca, Jacinto (2008). Finanzas Públicas. México: Editorial Porrua.

Instituto Nacional de Estadística y Geografía (2012). El bienestar subjetivo y la medición del progreso en las sociedades. Disponible en: http://www.inegi.org.mx/ inegi/contenidos/Investigacion/Experimentales/Bienestar/Presentacion.aspx

Instituto Nacional de Estadística y Geografía. Banco de Información Económica. Sistema de Cuentas Nacionales. Disponible en: http://www.inegi.org.mx/sistemas/ bie/

Instituto Nacional de Estadística y Geografía. Censo de Población y Vivienda 2010. Disponible en: http://www. inegi.org.mx/est/contenidos/proyectos/ccpv/cpv2010/ Default.aspx

Instituto Nacional de Estadística y Geografía. II Conteo de Población y Vivienda 2005. Disponible en: http:// www.inegi.org.mx/est/contenidos/proyectos/ccpv/ cpv2005/default.aspx

Lora, Eduardo (Coord.)(2008). Calidad de Vida. Mas allá de los hechos. México: Fondo de Cultura Económico Banco Interamericano de Desarrollo.

Observatorio de Política Social y Derechos Humanos. Ramo 33 Consolidado periodo 1998 - 2011. Disponible en http://www.observatoriopoliticasocial.org/index. php?option=com_content\&view=category\&id $=72$

Programa de las Naciones Unidas para el Desarrollo (2012a). El Índice de Desarrollo Humano en México: Cambios metodológicos e información para las entidades federativas. Disponible en: http://www.undp.org. $\mathrm{mx}$ /spip.php?page=publicacion\&id_article $=2338$

Programa de las Naciones Unidas para el Desarrollo (2012b). Informe sobre Desarrollo Humano México:
Equidad del gasto público: derechos sociales universales con subsidios focalizados. Disponible en: http://www. undp.org.mx/spip.php?page=area_interior\&id_rubrique $=120 \&$ id article $=1872 \&$ id $\_$parent $=119$

Programa de las Naciones Unidas para el Desarrollo (2012c). Informe sobre la Competitividad Social en México 2012. Disponible en: http://www.undp.org.mx/IMG/ pdf/Informe_sobre_Competitividad_Social_en_MexiCO_2012.pdf

Rojas, Mariano (Coord.)(2011). La medición del progreso y bienestar. Propuestas desde América Latina. México: Foro Consultivo Científico y Tecnológico A. C. Disponible en: http://www.foroconsultivo.org.mx/libros_editados/midiendo_el_progreso_2011_esp.pdf

Rojas, Mariano (Coord) (2011). Apuntes de la Conferencia Latinoamericana para la Medición del Bienestar y la Promoción del Progreso de las Sociedades. México: Foro Consultivo Científico y Tecnológico A. C. Disponible en: http://www.foroconsultivo.org.mx/libros_editados/ apuntes_conferencia_esp.pd

Rojas, Mariano y Martínez Ivan (Coord)(2012). Medición, Investigación e Incorporación a la Política Pública del Bienestar Subjetivo: América Latina. Reporte de la Comisión para el Estudio y la Promoción del Bienestar en América Latina. México: Foro Consultivo Científico y Tecnológico A. C. 2012. Disponible en: http://www.foroconsultivo.org.mx/libros_editados/midiendo_el_progreso_reporte_comision_esp.pdf

Sen, A., Stiglitz, J., Fitoussi, JP. (2009). Informe de la Comisión para la Medición del Desempeño Económico y Progreso Social. Disponible en: http://www.stiglitz-sen-fitoussi.fr/documents/rapport_anglais.pdf 